\title{
Application of a New Greyscale Descriptor for Recognition of Erythrocytes Extracted from Digital Microscopic Images
}

\author{
Dariusz Frejlichowski \\ West Pomeranian University of Technology, Szczecin \\ Faculty of Computer Science and Information Technology \\ Żołnierska 52, 71-210, Szczecin, Poland \\ dfrejlichowski@wi.zut.edu.pl
}

\begin{abstract}
In the paper an algorithm for description of greyscale objects extracted from images is applied for recognition of human red blood cells visible on digital microscopic images. This is a part of an approach for automatic (or semiautomatic) diagnosis of selected diseases based on the deformation of erythrocytes. The disease is concluded by means of the recognition of types of red blood cells visible on an digital microscopic image, stained using MGG method and converted into greyscale. The applied algorithm is based on the polar transform of pixels belonging to an object and a specialized method for constituting the resultant description, where derived coordinates are put into matrix, in which the row corresponds to the distance from the centre, and the column - to the angle. This assumption was previously applied only for shape features. The proposed algorithm includes several auxiliary steps, e.g. median and low-pass filtering in order to pre-process the extracted object in greyscale. The algorithm is experimentally evaluated and analysed. It is compared with four other greyscale descriptors, namely: ScaleInvariant Feature Transform, Gabor filter, Polar-Fourier Greyscale Descriptor, and the approach based on polar transform and projections.
\end{abstract}

\section{Keywords}

Image Recognition, Erythrocytes Identification, Greyscale Descriptor, Polar Transform

\section{INTRODUCTION}

In the paper a new algorithm for greyscale object description is applied to the problem of recognition of red blood cells extracted from digital microscopic images. The identification of particular types of erythrocytes is applied for the automatic (or semi-automatic) diagnosis of some selected diseases. It results from the fact that deformed erythrocytes cannot properly deliver oxygen. Hence, blood circulation is not regulated. Two exemplary diseases that can be concluded this way are anaemia and malaria. In both cases the appearance of cells is changed, what gives the possibility of performing the automatic analysis based on digital microscopic images using computer vision algorithms. In case of works described in the paper, the digital microscopic images stained by means of the MGG (MayGrunwald-Giemsa) method are applied. Considering the approaches applied for the problem, the template matching utilizing the greyscale as a feature is used.

\footnotetext{
Permission to make digital or hard copies of all or part of this work for personal or classroom use is granted without fee provided that copies are not made or distributed for profit or commercial advantage and that copies bear this notice and the full citation on the first page. To copy otherwise, or republish, to post on servers or to redistribute to lists, requires prior specific permission and/or a fee.
}

The template matching is a general approach in which the classes of objects for the identification are represented by templates stored in the template base. Usually one or a few templates represent a class. However, both templates and test objects are not stored in their original appearance. Instead, they are represented (described) using particular methods, so-called descriptors. These descriptors apply some low-level features, e.g. shape, texture, colour or greyscale. These algorithms are very popular nowadays, thanks to their fast and easy derivation [Ver15]. In the paper the greyscale as a feature is applied. This comes from the conclusion that in real world, greyscale can bring important and useful information, sometimes better than in case of other features [Fre19]. A new algorithm for greyscale object representation is used for the problem of red blood cells identification. It applies the polar transform of pixels and a method for deriving the description, where obtained new coordinates are put into matrix, in which the row corresponds to the distance from the centre of an object, and the column-to the angle. This proposition was taken from an algorithm for shape representation [Rau94] and was not used for the greyscale so far. The applied method includes several additional steps, e.g. median and low-pass filtering in order to preprocess the extracted object in greyscale. 
The proposed algorithm is applied in the problem of red blood cells recognition and the obtained results are described in this paper. However, in order to analyse the efficiency of the method, it is compared experimentally with four other greyscale descriptors: Scale-Invariant Feature Transform [Low04], Gabor filter [Kum18], Polar-Fourier Greyscale Descriptor [Fre11], and the approach based on polar transform and projections [Fre18].

The rest of the paper is organized as follows. The second section describes the related works, both by means of the erythrocytes analysis, and the greyscale applied as a feature. The third section provides the description of the algorithm prosed in the paper. The fourth section gives the basic information about the algorithms selected for the comparison with it. The fifth section presents the results of the experiment, and finally, the last section concludes the paper and gives some discussion about future plans.

\section{RELATED WORKS}

The usage of computer vision in the analysis of cells visible on the digital microscopic blood images is popular. However, various applications are taken into account, e.g. counting the cells without their identification (e.g. [Ven13]), analysis of all visible objects (e.g. [Ran07]), sometimes more complicated and hence less efficient. Even when only one type is taken into consideration, usually leukocytes are analysed (e.g. [Son02], [Sab04]). However, some progress in the examination of red blood cells was madeseveral algorithms were applied for this purpose so far, e.g. morphological operators [DiR02], threshold selection techniques [Ros06], histogram [Dia09], deformable templates [Bro00], and polar-logarithmic transform [Lue05]. In [Fre10] three shape descriptors based on polar transform were investigated, namely Log-Pol-Fourier, UNL-Fourier and Point Distance Histogram. Very popular lately Deep Convolutional Neural Networks were applied to the problem of automatic identification of malaria infected erythrocytes [Don07]. Usually, for the recognition of red blood cells the shape is applied as the feature representing an object. However, the greyscale is also applicable (e.g. [Fre11], [Fre18], [Fre19]). This feature is less popular than other ones. Additionally, the algorithms are usually utilized for the whole image, yet they can be applied for the extracted object as well. In this manner, Scale-Invariant Feature Transform (SIFT) is especially popular [Low04]. A modification of this method (so-called extended SIFT) was also proposed [KeY04]. The Speeded Up Robust Features (SURF) approach is a second popular algorithm designed for this goal [Bay08]. Similarly, the Scale-Invariant Shape Features (SISF) were utilized in the problem of detection based on greyscale [Jur04]. On the other hand, human detection was also an area of interest, and for this task the Histograms of Oriented Gradients (HoG) were applied [Dal05]. The histogram for a scene in greyscale was also used [Chi11].

Texture analysis can be in some application very close to the usage of greyscale descriptors. Some examples of texture descriptors that could be applied in that way are: Local Binary Pattern with Local Phase Quantization [Nan16a], connectivity indexes in local neighbourhoods [Flo16a], Gaussian Markov Random Fields [Dha14], Gabor features [Kum18], non-uniform patterns [Nan16b], genetic algorithms [Wan17], local fractal dimensions [Flo16b], Fisher tensors with 'bag-of-words' [Far14], and approach based on morphology [Apt11].

\section{PROPOSED APPROACH}

The algorithm described in this paper is based on the idea given by T. W. Rauber and A. S. Steiger-Garcao for shape representation [Rau94]. Proposed by them shape representation, called UNL (named after the Universidade Nova de Lisboa), was based on the assumption that derived polar coordinates for silhouette points were put into a matrix, in which the row corresponds to the distance from centroid, and the column-to the angle. The difference in the resultant representation from the traditional polar transform for a shape can be easily noticed-see Figure 1.

In this paper above-mentioned idea is extended to the greyscale object representation. An example of a greyscale object and its transformed representation by means of the proposed approach is given in Figure 2.

The proposed algorithm is similar to the Polar-Fourier Greyscale Descriptor, proposed in [Fre11]. The differ-

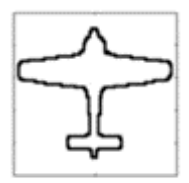

a

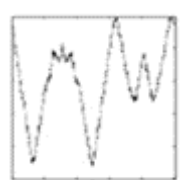

b

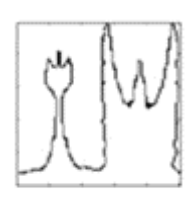

c
Figure 1: The difference in shape representation for object (a) between polar transform (b) and UNLtransform (c).
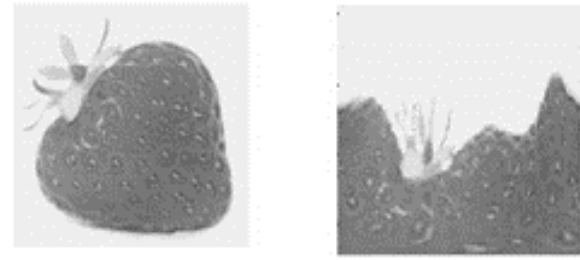

Figure 2: The result of the transformed greyscale object by means of the proposed approach proposed: original object on the left and its transformed representation on the right. 
ence between the two approaches is explained in Section 4.3.

Similarly to the Polar-Fourier Greyscale Descriptor, the proposed algorithm applies some additional pre-processing steps, improving the obtained representation, namely: median filtering, low-pass filtering, the construction of the constant rectangle containing the object, filling the gaps with the background colour, and resizing the transformed image to the constant size.

The algorithm for computing the resultant greyscale object representation is given in details below.

Step 1. Median filtering of the input sub-image $I$, with the kernel size 3.

Step 2. Low-pass filtering, realized through the convolution with mask $3 \times 3$ pixels, and normalization parameter equal to 9 .

Step 3. Calculation of the centroid by means of the moments [Hup95]. Firstly, $m_{00}, m_{10}, m_{01}$ are derived, and then the centroid $O=\left(x_{c}, y_{c}\right)$ :

$$
\begin{aligned}
& m_{p q}=\sum_{x} \sum_{y} x^{p} y^{q} I(x, y), \\
& x_{c}=\frac{m_{10}}{m_{00}}, \quad y_{c}=\frac{m_{01}}{m_{00}} .
\end{aligned}
$$

Step 4. Finding the maximal distances $d_{\max X}, d_{\max Y}$ for $X$ - and $Y$-axis respectively from the boundaries of $I$ to $O$.

Step 5. Expanding the image into both directions by $d_{\max X}-x_{c}$ and $d_{\max Y}-y_{c}$ and filling in the new parts using constant greyscale level, e.g. 127.

Step 6. Derivation of new coordinates and insertion in the image $P$, in which the row corresponds to the distance from centroid $\left(\rho_{i}\right)$, and the column - to the angle $\left(\theta_{i}\right)$ :

$$
\begin{gathered}
\rho_{i}=\sqrt{\left(x_{i}-x_{c}\right)^{2}+\left(y_{i}-y_{c}\right)^{2}}, \\
\theta_{i}=\operatorname{atan}\left(\frac{y_{i}-y_{c}}{x_{i}-x_{c}}\right) .
\end{gathered}
$$

Step 7. Resizing $P$ to the constant rectangular size, $n \times n$, e.g. $n=128$.

Step 8. Derivation of 2D Fourier transform:

$$
\begin{array}{r}
C(k, l)=\frac{1}{H W} \mid \sum_{h=1}^{H} \sum_{w=1}^{W} P(h, w) . \\
\exp ^{\left(-i \frac{2 \pi}{H}(k-1)(h-1)\right)} \exp ^{\left(-i \frac{2 \pi}{W}(l-1)(w-1)\right)} \mid,
\end{array}
$$

where:

$H, W$-height and width of $P$,

$k$-sampling rate in vertical direction $(k \geq 1$ and $k \leq H$ ),

$l$-sampling rate in horizontal direction $(l \geq 1$ and $l \leq W)$,

$C(k, l)$ - the coefficient of discrete Fourier transform in $k$-th row and $l$-th column,

$P(h, w)$ - value in the resultant image plane with coordinates $h, w$.

Step 9. Selection of the spectrum sub-part, e.g. $10 \times 10$ size and concatenation into vector $V$.

\section{THE ALGORITHMS APPLIED FOR THE EXPERIMENTAL COMPARI- SON}

The proposed algorithm, described in the previous section, was compared and experimentally investigated with several other algorithms for greyscale object representation. The selection was based on an assumption that a popular method for object localization in greyscale images and the one popular for texture representation should be evaluated. Both are applied in object description as well. Also, two previous algorithms for greyscale objects representation proposed by the Author were used. That gave four algorithms that were compared with the proposition given in the paper. Their description is given briefly in the following subsections.

\subsection{Scale-Invariant Feature Transform}

The Scale-Invariant Feature Transform (SIFT) was proposed by D. G. Lowe [Low04], [Low11]. The description obtained using it is invariant to rotation and scaling. The algorithm is very popular as a method for feature detection. However, it can be easily applied as a representation of an object in greyscale. Therefore, in works described in this paper it is utilized that way. Moreover, some approaches were proposed in order to speed-up the derivation of the representation [Hey07]

\subsection{The Gabor descriptor}

The Gabor filter is mainly used as a texture descriptor [Zha09]. However, its practical usefulness in representing the grey levels lead to its applicability also in a form of a greyscale descriptor. Similarly to the previously mentioned algorithm, the Gabor filter is applied in the experiments described in the following section.

\subsection{The Polar-Fourier Greyscale Descrip- tor}

The Polar-Fourier Greyscale Descriptor was proposed in [Fre11] and is in fact a source for the descriptor ap- 
plied in this paper. Hence, it is very similar to the algorithm described in the previous section. The significant difference occurs in Step 6. In the original PolarFourier Greyscale Descriptor simple polar transform is applied. The proposed in this paper approach is similarly to the UNL-transform more sophisticated, what was explained in the introductory part of Section 3.

\subsection{The Descriptor based on Polar Trans- form and Vertical and Horizontal Pro- jections}

The approach applying the polar transform and projections has similar initial stages as the Polar-Fourier Greyscale Descriptor, however the rest is different, since instead of Fourier transform the vertical and horizontal projections are applied. The whole descriptor is given as follows [Fre18]:

Step 1. Median filtering of the input image $I$, with the kernel size 3 .

Step 2. Low-pass filtering, realized through the convolution with mask $3 \times 3$ pixels, and normalization parameter equal to 9 .

Step 3. Calculation of the centroid by means of the moments [Hup95]. Firstly $m_{00}, m_{10}, m_{01}$ are derived, and later the centroid $O=\left(x_{c}, y_{c}\right)$ :

$$
\begin{aligned}
& m_{p q}=\sum_{x} \sum_{y} x^{p} y^{q} I(x, y), \\
& x_{c}=\frac{m_{10}}{m_{00}}, \quad y_{c}=\frac{m_{01}}{m_{00}} .
\end{aligned}
$$

Step 4. Transforming $I$ into polar coordinates (resultant image is denoted as $P$ ), by means of the formulas:

$$
\begin{gathered}
\rho_{i}=\sqrt{\left(x_{i}-x_{c}\right)^{2}+\left(y_{i}-y_{c}\right)^{2}}, \\
\theta_{i}=\operatorname{atan}\left(\frac{y_{i}-y_{c}}{x_{i}-x_{c}}\right) .
\end{gathered}
$$

Step 5. Resizing $P$ to the constant rectangular size, $n \times n$, e.g. $n=128$.

Step 6. Deriving the horizontal and vertical projections of $P$ :

$$
H_{i}=\sum_{j=1}^{n} P_{i, j}, \quad V_{j}=\sum_{i=1}^{n} P_{i, j} .
$$

Step 7. Concatenating the obtained vectors $H$ and $V$ into one, $C=H V$, representing an object.

\section{EXPERIMENTAL RESULTS}

Before the representation and identification of particular red blood cells, they have to be segmented and extracted. For this purpose the approach proposed in [Fre10] was applied. It starts with the conversion of the input image into greyscale. Later the modified histogram thresholding is applied in order to obtain the binary image. Then, particular objects can be localized. This process is performed by tracing regions of each separate objects. Only objects entirely placed within a processed image are considered. The area of extracted regions is analysed in order to reject thrombocytes and leukocytes. In order to limit the processed objects to erythrocytes, if a region is larger (for leukocytes) or smaller (for thrombocytes) then it is rejected (the difference in size for these three main objects of interest is provided in Figure 3). This assumption results from the fact that leukocytes (e.g. monocytes) have the size of 40 $\mu m$, thrombocytes - ca. $2 \mu m$, and the size of erythrocytes varies between 6 and $12 \mu \mathrm{m}$. Additionally, this process rejects some occluded objects, what is a benefit, since they are difficult to recognise. Nevertheless, it is still possible that some undesirable objects remain, if their area is similar to erythrocytes. In order to avoid this problem, the analysis of object's histogram is applied, since this feature varies for different particlesthrombocytes and leukocytes have almost black parts inside. Moreover, the histogram equalisation of the image before the binarisation is performed, what reduces the number of occluded shapes.

As a result of the above-described, by means of using the established coordinates of a single cell, the rectangular subpart of the greyscale image with it is extracted and processed in next stages.

The experiments were performed using 55 MayGrunwald-Giemsa stained microscopic images magnified 1,000 times. All of them were converted into greyscale, and then every cell was localised and extracted separately by means of the above-described approach. For each object the proposed algorithm as

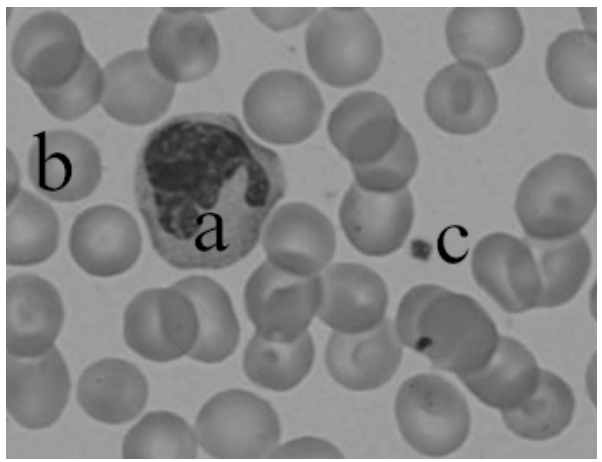

Figure 3: Three different types of human blood cells, varying in size: a) leukocyte, b) erythrocyte, c) thrombocyte [Omi14]. 
well as the algorithms described in the previous section were applied in order to obtain the descriptions. They were matched using the dissimilarity measure with the stored templates that were represented in the same way. Twelve classes of erythrocytes were applied. For each class five template objects were used. That gave in result 60 templates, since 12 classes of erythrocytes were considered.

The number of cells within a single MGG image varies significantly. In case of the images applied for the experiments, this number varies from a dozen to more than a hundred. In order to emphasize the number of analysed and processed objects, some statistical information will be given. It is limited to the number of extracted erythrocytes in the most limited case. After the rejection of larger (leukocytes and occluded cells) and smaller (thrombocytes) objects the overall number of processed objects of interest was equal to 2,772. The average for an image was (rounded) 50, and the median value was 44 . The smallest number of properly extracted erythrocytes was equal to 10 objects, and the highest was 102 .

The obtained efficiency for particular erythrocyte types and greyscale descriptors applied in the experiment is given in Table 1. Additionally, in the table average efficiency for all analysed descriptors is provided.

\section{CONCLUDING REMARKS AND FU- TURE PLANS}

In the paper an algorithm for description of greyscale objects extracted from digital images was applied for recognition of human red blood cells visible on digital microscopic images. This is a part of an approach for automatic (or semi-automatic) diagnosis of selected diseases based on the deformation of erythrocytes. The disease is concluded by means of the recognition of types of red blood cells visible on a digital microscopic image, stained using MGG method and converted into greyscale.

The proposed algorithm was experimentally investigated in comparison with four other greyscale descriptors. It obtained the best results by means of the efficiency in recognizing the types of erythrocytes.

Nevertheless, future works are planned on further improvement in the representation of objects extracted from digital images and represented using greyscale as a feature. Another approaches are planned to be analysed.

Moreover, since the representation of cells was the main topic of the works described in this paper, more attention should be put on the classification stage. Here, the simplest method based on the template matching was applied. It was assumed that some more sophisticated and novel approaches should provide even better overall results. Hence, the second direction of research should be related to the analysis of the methods belonging to the last steps of the method. For example, application of some deep learning algorithms, very popular nowadays, would definitely lead to better results.

Finally, the Electron Microscopy seems to be a tempting object of future works. The tasks would be slightly different in that case, e.g. the rejection of erythrocytes could be performed in order to pre-process an image for other applications. However, since objects visible on EM images are spatial, some significant modifications of the proposed description algorithm should be made.

\section{REFERENCES}

[Apt11] Aptoula, E., Lefèvre, S.: Morphological texture description of grey-scale and color images. Advances in Imaging and Electron Physics, Vol. 169, pp. 1-74, 2011.

[Bay08] Bay, H., Ess, A., Tuytelaars, T., Van Gool, L. SURF: Speeded Up Robust Features. Computer Vision and Image Understanding, Vol. 110, Iss. 3, pp. 346-359, 2008.

[Bro00] Bronkorsta, P.J.H., et al. On-line detection of red blood cell shape using deformable templates. Pattern Recognition Letters, Vol. 21, Iss. 5 , pp. 413-424, 2000.

[Chi11] Chin, T.J., Suter, D., Wang, H. Boosting histograms of descriptor distances for scalable multiclass specific scene recognition. Image and Vision Computing, Vol. 29, Iss. 4, pp. 241-250, 2011.

[Dal05] Dalal, N., Triggs, B. Histograms of oriented gradients for human detection. IEEE Computer Society Conference on Computer Vision and Pattern Recognition (CVPR 2005), Vol. 1, pp. 886893, 2005.

[Dha14] Dharmagunawardhana, C., Mahmoodi, S., Bennett, M., Niranjan, M. Gaussian Markov random field based improved texture descriptor for image segmentation. Image and Vision Computing, Vol. 32, Iss. 11, pp. 884-895, 2014.

[Dia09] Diaz, G., Gonzalez, F. A., Romero, E. A semiautomatic method for quantification and classification of erythrocytes infected with malaria parasites in microscopic images. Journal of Biomedical Informatics, Vol. 42, Iss. 2, pp. 296-307, 2009.

[DiR02] Di Ruberto C, Dempster A, Khan S, Jarra B. Analysis of infected blood cell images using morphological operators. Image and Vision Computing, Vol. 20, pp. 133-146, 2002.

[Don07] Dong, Y. et al., Evaluations of deep convolutional neural networks for automatic identification 


\begin{tabular}{|c|c|c|c|c|c|}
\hline & SIFT & Gabor & P-F GD & $\begin{array}{c}\text { polar transform } \\
\text { and projections }\end{array}$ & $\begin{array}{c}\text { proposed } \\
\text { descriptor }\end{array}$ \\
\hline schistocyte & $85 \%$ & $75 \%$ & $69 \%$ & $61 \%$ & $92 \%$ \\
\hline dacrocyte & $93 \%$ & $86 \%$ & $83 \%$ & $72 \%$ & $95 \%$ \\
\hline acantocyte & $98 \%$ & $94 \%$ & $98 \%$ & $89 \%$ & $100 \%$ \\
\hline echinocyte & $96 \%$ & $81 \%$ & $89 \%$ & $88 \%$ & $95 \%$ \\
\hline ovalocyte & $78 \%$ & $70 \%$ & $74 \%$ & $69 \%$ & $86 \%$ \\
\hline normocyte & $94 \%$ & $95 \%$ & $97 \%$ & $86 \%$ & $100 \%$ \\
\hline stomatocyte & $90 \%$ & $87 \%$ & $90 \%$ & $83 \%$ & $98 \%$ \\
\hline codocyte & $80 \%$ & $76 \%$ & $72 \%$ & $65 \%$ & $80 \%$ \\
\hline spherocyte & $100 \%$ & $98 \%$ & $100 \%$ & $93 \%$ & $100 \%$ \\
\hline leptocyte & $100 \%$ & $98 \%$ & $97 \%$ & $91 \%$ & $100 \%$ \\
\hline annular eryth. & $82 \%$ & $80 \%$ & $76 \%$ & $64 \%$ & $84 \%$ \\
\hline drepanocyte & $92 \%$ & $78 \%$ & $81 \%$ & $74 \%$ & $90 \%$ \\
\hline average & $\mathbf{9 1 \%}$ & $\mathbf{8 5 \%}$ & $\mathbf{8 6 \%}$ & $\mathbf{7 8 \%}$ & $\mathbf{9 3 \%}$ \\
\hline
\end{tabular}

Table 1: Average recognition rates (efficiency) obtained for particular classes of erythrocytes by means of experimentally investigated greyscale descriptors.

of malaria infected cells. 2017 IEEE EMBS International Conference on Biomedical \& Health Informatics (BHI), Orlando, FL, pp. 101-104, 2017.

[Far14] Faraki, M., Harandi, M.T., Wiliem, A., Lovell, B.C. Fisher tensors for classifying human epithelial cells. Pattern Recognition, Vol. 47, Iss. 7, pp. 2348-2359, 2014.

[Flo16a] Florindo, J.B., Landini, G., Bruno, O.M. Three-dimensional connectivity index for texture recognition. Pattern Recognition Letters, Vol. 84, pp. 239-244, 2016.

[Flo16b] Florindo, J.B., Bruno, O.M. Local fractal dimension and binary patterns in texture recognition. Pattern Recognition Letters, Vol. 78, pp. 22 27, 2016.

[Fre10] Frejlichowski D. Pre-processing, extraction and recognition of binary erythrocyte shapes for computer-assisted diagnosis based on MGG images. In: Bolc, L. et al. (eds.): ICCVG 2010, Part I. Lecture Notes in Computer Science, Vol. 6374, pp. 368-375, 2010.

[Fre11] Frejlichowski, D. Identification of erythrocyte types in greyscale MGG images for computerassisted diagnosis. IbPRIA 2011, Lecture Notes in Computer Science, Vol. 6669, pp. 636-643, 2011.

[Fre18] Frejlichowski, D. A new algorithm for greyscale objects representation by means of the polar transform and vertical and horizontal pro- jections. ACIIDS 2018: Intelligent Infor-mation and Database Systems, Lecture Notes in Artifical Intelligence, Vol. 10752, pp. 617-625, 2018.

[Fre19] Frejlichowski, D. Low-Level Greyscale Image Descriptors Applied for Intelligent and Contextual Approaches. In: Nguyen N., Gaol F., Hong TP., Trawiński B. (eds) Intelligent Information and Database Systems. ACIIDS 2019. Lecture Notes in Computer Science, Vol. 11432, pp. 441451, 2019.

[Hey07] Heymann, S. Maller, K., Smolic, A., Froehlich, B. and Wiegand, T. SIFT implementation and optimization for general-purpose GPU. Proceedings of the International Conference in Central Europe on Computer Graphics, Visualization and Computer Vision, pp. 317-322, 2007.

[Hup95] Hupkens, T.M., de Clippeleir, J. Noise and intensity invariant moments. Pattern Recognition Letters, Vol. 16, Iss. 4, pp. 371-376, 1995.

[Jur04] Jurie, F., Schmid, C. Scale-invariant shape features for recognition of object categories. 2004 IEEE Computer Society Conference on Computer Vision and Pattern Recognition (CVPR 2004), Vol. 2, II-90-II-96, 2004.

[KeY04] Ke, Y., Sukthankar, R. PCA-SIFT: a more distinctive representation for local image descriptors. 2004 IEEE Computer Society Conference on Computer Vision and Pattern Recognition (CVPR 2004), Vol. 2, II-506-II-513, 2004.

[Kum18] Kumar, M., Singh, Kh. M. Retrieval of head- 
neck medical images using Gabor filter based on power-law transformation method and rank BHMT. Signal, Image and Video Processing, Vol. 12, Iss. 5, pp. 827-833, 2018.

[Low04] Lowe, D. G. Distinctive Image Features from Scale-Invariant Keypoints, International Journal of Computer Vision, Vol. 60, Iss. 2, pp. 91-110, 2004.

[Low11] Low, Y.-Q., Lee, S.-W., Goi, B.-M., and Ng, M.-S. A New SIFT-based camera calibration method for hybrid dual-camera. In: A. Abd Manaf et al. (Eds.): ICIEIS 2011, Part II, CCIS, Vol. 252, pp. 96-103, 2011.

[Lue05] Luengo-Oroz, M. A., Angulo, J., Flandrin, G., Klossa, J. Mathematical morphology in polarlogarithmic coordinates. Application to erythrocyte shape analysis. In: Marques, J. S., Perez de la Blanca, N., Pina, P. (eds.) IbPRIA 2005. Lecture Notes in Computer Science, Vol. 3523, pp. 199205, 2005.

[Nan16a] Nanni, L., Melucci, M. Combination of projectors, standard texture descriptors and bag of features for classifying images. Neurocomputing, Vol. 173, pp. 1602-1614, 2016.

[Nan16b] Nanni, L., Brahnam, S., Lumini, A. A simple method for improving local binary patterns by considering non-uniform patterns. Pattern Recognition, Vol. 45, Iss. 10, pp. 3844-3852, 2012.

[Omi14] Omid, S., Rabbani, H., Talebi, A., and Banaem, H.U. Selection of the best features for leukocytes classification in blood smear microscopic images. In SPIE Medical Imaging, pp. 90410P. International Society for Optics and Photonics, 2014.

[Ran07] Ranzato, M. et al. Automatic recognition of biological particles in microscopic images. Pattern Recognition Letters, Vol. 28, Iss. 1, pp. 3139, 2007.

[Rau94] Rauber, T. Two-dimensional shape description. Technical report, University Nova de Lisboa, Portugal, 1994.

[Ros06] Ross, N.E., Pritchard, C.J., Rubin, D.M. et al. Automated image processing method for the diagnosis and classification of malaria on thin blood smears. Medical and Biological Engineering and Computing, Vol. 44, pp. 427-436, 2006.

[Sab04] Sabino, D. M. U., da Fontoura Costa, L., Rizzatti, E. G., Zago, A. M. A texture approach to leukocyte recognition. Real-Time Imaging, Vol. 10, Iss. 4, pp. 205-216, 2004.

[Son02] Song, X. B., Abu-Mostafa, Y., Sill, J., Kasdan, H., Pavel, M. Robust image recognition by fusion of contextual information. Information $\mathrm{Fu}$ sion, Vol. 3, Iss. 4, pp. 277-287, 2002.
[Ven13] Venkatalakshmi, B. and Thilagavathi, K. Automatic red blood cell counting using Hough transform. 2013 IEEE Conference on Information \& Communication Technologies, Thuckalay, Tamil Nadu, India, pp. 267-271, 2013.

[Ver15] Verma, M., Raman, B. Center symmetric local binary co-occurrence pattern for texture, face and bio-medical image retrieval. Journal of Visual Communication and Image Represen-tation, No. 32, pp. 224-236, 2015.

[Wan17] Wang, S. et al. Texture analysis method based on Fractional Fourier Entropy and Fitness-scaling Adaptive Genetic Algorithm for detecting leftsided and right-sided sensorineural hearing loss. Fundamenta Informaticae, Vol. 151, Iss. 1-4, pp. 505-521, 2017.

[Zha09] Zhan, X., Xingbo, S., Yuerong L. Comparison of two gabor texture descriptor for texture classification. In: 2009 WASE International Conference on Information Engineering, pp. 52-56, Taiyuan, Chanxi, 2009. 